\title{
Total energy equation leading to exchange-correlation functional
}

\author{
LIU Feng \& WANG TzuChiang* \\ State Key Laboratory of Nonlinear Mechanics, Institute of Mechanics, Chinese Academy of Sciences, Beijing 100190, China
}

Received September 25, 2014; accepted December 17, 2014; published online January 14, 2015

\begin{abstract}
By solving the total energy equation, we obtain the formula of exchange-correlation functional for the first time. This functional is usually determined by fitting experimental data or the numerical results of models. In the uniform electron gas limit, our exchangecorrelation functional can exactly reproduce the results of Perdew-Zunger parameterization from the jellium model. By making use of a particular solution, our exchange-correlation functional could take into account the case of non-uniform electron density, and its validity can be confirmed through comparisons of the band structure, equilibrium lattice constant, and bulk modulus of aluminum and silicon. The absence of mechanical prescriptions for the systematic improvement of exchange-correlation functional hinders further development of density-functional theory (DFT), and the formula of exchange-correlation functional given in this study might provide a new perspective to help DFT out of this awkward situation.
\end{abstract}

Density-functional theory, exchange-correlation functional, bulk modulus, band structure, aluminum, silicon

PACS number(s): $31.15 . \mathrm{Ew}, 71.15 . \mathrm{Mb}, 71.15 . \mathrm{Nc}, 71.20 . \mathrm{b}$

Citation: Liu F, Wang T C. Total energy equation leading to exchange-correlation functional. Sci China-Phys Mech Astron, 2015, 58: 054601, doi: 10.1007/s11433-014-5639-2

\section{Introduction}

Although density-functional theory (DFT) was proposed back in 1964 [1,2] and was popular for calculating periodic system in 1970s, it was seldom applied to quantum chemistry until the development of exchange-correlation functionals in 1990s, which helped it win the 1998 Nobel Prize in chemistry. There are much richer applications for DFT now, for example, simulations of clusters, catalysis, and nanotechnology, which has been stimulated by the progress in approximations for the exchange-correlation functional [3-16], since it is the only unknown in this theory.

Perdew et al. [17] considered exchange-correlation functional approximations as a Jacob's ladder, and they classified them into five rungs based on the ingredients used to construct the exchange-correlation functional. The five rungs from the bottom up are the local spin density approximation, generalized gradient approximation (GGA), meta-

*Corresponding author (email: tcwang@imech.ac.cn) gradient approximation, exact exchange and compatible correlation and exact exchange and exact partial correlation. The Jacob's ladder rises to the goal of "chemical accuracy", which means the rates of chemical reactions (the order of $1 \mathrm{kcal} / \mathrm{mol}$ $=0.0434 \mathrm{eV}$ ) can be well predicted. With the exact exchangecorrelation functional DFT will be the ultimate practical theory, and it could be almost perfect if there exists any mechanical prescription that would systematically improve approximations [18]. Unfortunately, neither possibility exits at the moment. Standing on the middle rung of Jacob's ladder, we can-not see the next rung. With more research on physical models and also constraint satisfaction [19-28], the nature of exchange-correlation functional will be gradually uncovered. However, this research can hardly help design a better exchange-correlation functional, and we believe that this is because these models or constraints can-not suggest the form of exchange-correlation functional directly. Consequently, one is naturally led to the question, "Is there any rational consideration for searching the formula of exchange- 
correlation functional?"

In this paper, we obtain the formula of exchangecorrelation functional by solving the total energy equation. In the uniform electron gas (UEG) limit, our exchangecorrelation functional can exactly reproduce the results of Perdew-Zunger (PZ/CA) parameterization from the jellium model. For the case of non-uniform electron density, the contribution of the particular solution of the total energy equation is considered, and comparisons of the equilibrium lattice constant, bulk modulus and band structure of aluminum and silicon support its validity. This study, we believe, could provide a new perspective to help DFT out of the present impasse.

\section{Formulism}

Based on the Born-Oppenheimer approximation, the motions of electrons and nuclei can be decoupled, which means that in order to study electrons we only need to focus on the energy relevant to electrons. To be specific

$$
E_{T}=T+V_{e e}+\int v_{e x t}(\boldsymbol{r}) \rho(\boldsymbol{r}) \mathrm{d} \boldsymbol{r}
$$

where $T, V_{e e}$, and $v_{e x t}(\boldsymbol{r})$ represent the kinetic energy of electrons, the interaction energy between electrons and the external potential, respectively. In the Kohn-Sham method, the energy functional can be written as:

$$
\begin{aligned}
& E_{T}[\rho]=T_{s}[\rho]+V_{H}[\rho]+E_{x c}[\rho]+\int v_{e x t}(\boldsymbol{r}) \rho(\boldsymbol{r}) \mathrm{d} \boldsymbol{r}, \\
& V_{H}[\rho]=\frac{1}{2} \int \frac{\rho\left(\boldsymbol{r}^{\prime}\right) \rho(\boldsymbol{r})}{\left|\boldsymbol{r}-\boldsymbol{r}^{\prime}\right|} \mathrm{d} \boldsymbol{r}^{\prime} \mathrm{d} \boldsymbol{r}, \\
& E_{x c}[\rho] \equiv T[\rho]-T_{s}[\rho]+V_{e e}[\rho]-V_{H}[\rho],
\end{aligned}
$$

where $T_{s}[\rho]$ is the single particle kinetic energy, $V_{H}[\rho]$ is the classical Coulomb energy, or Hartree energy, and $E_{x c}$ is the exchange-correlation energy. Using variational principle proposed in the second Hohenberg-Kohn theorem, the wellknown single particle equation, the Kohn-Sham equation, can be obtained

$$
\begin{aligned}
& {\left[-\frac{1}{2} \nabla^{2}+v_{e f f}\right] \phi_{i}=\epsilon_{i} \phi_{i},} \\
& V_{e f f}(\boldsymbol{r})=v_{e x t}(\boldsymbol{r})+\int \frac{\rho\left(\boldsymbol{r}^{\prime}\right)}{\left|\boldsymbol{r}-\boldsymbol{r}^{\prime}\right|} \mathrm{d} \boldsymbol{r}^{\prime}+v_{x c}(\boldsymbol{r}),
\end{aligned}
$$

where $v_{x c}(\boldsymbol{r})=\delta E_{x c} / \delta \rho(\boldsymbol{r})=\rho(\boldsymbol{r}) \cdot \delta \epsilon_{x c} / \delta \rho(\boldsymbol{r})+\epsilon_{x c}$ and $\epsilon_{x c}$ are the exchange-correlation potential and its energy density. By using the energy eigenvalues $\epsilon_{i}$ obtained from the KohnSham equation, the energy functional can be rewritten as:

$$
E_{T}[\rho]=\sum_{i}^{N} \epsilon_{i}-V_{H}[\rho]+E_{x c}[\rho]-\int v_{x c}(\boldsymbol{r}) \rho(\boldsymbol{r}) \mathrm{d} \boldsymbol{r} .
$$

Here $N$ is the total number of electrons in the system. Note that eq. (7) is the starting point of this paper. Usually, eq. (7) is considered as a formula to calculate the total energy of the system. However, we will reconsider it as an equation (given the external potential and also the total number of electrons, the energy of the ground state is already determined, which means $E_{g}=E\left(\rho_{g}\right)=\sum_{i}^{N} \epsilon_{i}-V_{H}\left[\rho_{g}\right]+E_{x c}\left[\rho_{g}\right]-\int v_{x c} \rho_{g}(\boldsymbol{r}) \mathrm{d} \boldsymbol{r}$. Since the external potential $V_{\text {ext }}$ could have any form (so does the corresponding $\rho_{g}(\boldsymbol{r})$ ), and also considering the universality of the exchange-correlation functional, the above equation can be built unambiguously. This is why we can consider it as an equation for the exchange-correlation functional. Note that $E_{g}$ is a parameter dependent of the given system (i.e., $V_{\text {ext }}$ ) in our formalism.), which must be satisfied by $\epsilon_{x c}[\rho]$ (since $v_{x c}[\rho]$ can be expressed by $\epsilon_{x c}[\rho]$, as mentioned above). Actually, eq. (7) provides constraints for the exchange-correlation functional, which is constructive when searching for its formula. Thus, we will next carefully discuss the solution of eq. (7). First, $v_{x c}=\rho \cdot \delta \epsilon_{x c} / \delta \rho+\epsilon_{x c}$ is used to simplify eq. (7)

$$
-\int \frac{\delta \epsilon_{x c}[\rho]}{\delta \rho(\boldsymbol{r})} \rho(\boldsymbol{r}) \rho(\boldsymbol{r}) \mathrm{d} \boldsymbol{r}=E_{T}-\sum_{i}^{N} \epsilon_{i}+V_{H}[\rho] .
$$

Eq. (8) is an integral-differential equation for unknown function $\epsilon_{x c}$. Due to the superposability for a linear equation, the solution of eq. (8) can be divided into two parts $\epsilon_{x c}[\rho]=\epsilon_{x c}^{(H)}[\rho]+\epsilon_{x c}^{(S)}[\rho]$, i.e., the homogeneous solution $\epsilon_{x c}^{(H)}[\rho]$ and the particular solution $\epsilon_{x c}^{(S)}[\rho]$, which satisfy

$$
\begin{aligned}
& -\frac{\delta \epsilon_{x c}^{(S)}[\rho]}{\delta \rho(\boldsymbol{r})} \rho(\boldsymbol{r})=\frac{1}{N}\left[E_{T}-\sum_{i}^{N} \epsilon_{i}\right]+\frac{1}{2} \int \frac{\rho\left(\boldsymbol{r}^{\prime}\right)}{\left|\boldsymbol{r}-\boldsymbol{r}^{\prime}\right|} \mathrm{d} \boldsymbol{r}^{\prime}, \\
& \int \frac{\delta \epsilon_{x c}^{(H)}[\rho]}{\delta \rho(\boldsymbol{r})} \rho(\boldsymbol{r}) \rho(\boldsymbol{r}) \mathrm{d} \boldsymbol{r}=0 .
\end{aligned}
$$

\subsection{Particular solution $\epsilon_{x c}^{(S)}[\rho]$}

It seems easy to obtain the particular solution

$$
\begin{aligned}
& \epsilon_{x c}^{(S)}[\rho]=-\frac{\Delta E}{N} \ln \frac{\rho}{\rho}-\frac{1}{2} \int \frac{U(\boldsymbol{r})}{\rho} \mathrm{d} \rho, \\
& \Delta E=E_{T}-\sum_{i}^{N} \epsilon_{i}, \\
& U(\boldsymbol{r})=\int \frac{\rho(\boldsymbol{r})}{\left|\boldsymbol{r}-\boldsymbol{r}^{\prime}\right|} \mathrm{d} \boldsymbol{r}^{\prime},
\end{aligned}
$$

where $\bar{\rho}$ represents the average electron density. Note that $U(\boldsymbol{r})$ in above formula is the Coulomb repulsion potential between electrons. Because $U(\boldsymbol{r})$ is a long-range interaction, it will diverge under periodic boundary conditions, which are usually assumed for calculations of crystals. In fact, this divergence originates from non-neutrality, which can be understood by writing Poisson's equation

$$
\Delta U(\boldsymbol{r})=4 \pi \rho(\boldsymbol{r}) .
$$

Note that the above equation only considers electrons. Under periodic boundary conditions, the solution in real space is 
not easy, but fortunately in reciprocal space Poisson's equation becomes diagonal, making the solution, in principle, trivial,

$$
|\boldsymbol{G}|^{2} U(\boldsymbol{G})=4 \pi \rho(\boldsymbol{G})
$$

Now we focus on the term $U(\boldsymbol{G}=0)$. Usually, Poisson's equation considers nuclei and electrons all at once, and the neutrality of the system confirms $\rho(\boldsymbol{G}=0)=0$, indicating that $U(\boldsymbol{G}=0)$ is an arbitrary constant, and can be left alone. However, the situation here is different, since only electrons are considered in Poisson's equation, $\rho(\boldsymbol{G}=0) \neq 0$. The consequence of this non-neutrality is that $U(\boldsymbol{G}=0)$ diverges. To deal with it, the $U(\boldsymbol{r})$ is divided into two components

$$
\begin{aligned}
& U(\boldsymbol{r})=U_{0}(\boldsymbol{r})+U_{*}(\boldsymbol{r}), \\
& \Delta U_{0}(\boldsymbol{r})=4 \pi \bar{\rho}, \\
& \Delta U_{*}(\boldsymbol{r})=4 \pi[\rho(\boldsymbol{r})-\bar{\rho}] .
\end{aligned}
$$

Now the particular solution can be given as:

$$
\begin{aligned}
& \epsilon_{x c}^{\left(S_{1}\right)}[\rho]=-\frac{E^{\prime}}{N} \ln \frac{\rho}{\bar{\rho}}-\frac{1}{2} \int \frac{U_{*}(\boldsymbol{r})}{\rho} \mathrm{d} \rho, \\
& E^{\prime}=E_{T}-\sum_{i}^{N} \epsilon_{i}+E_{0}, \\
& E_{0}=\frac{1}{2} \int U_{0}(\boldsymbol{r}) \rho(\boldsymbol{r}) \mathrm{d} \boldsymbol{r},
\end{aligned}
$$

and the corresponding exchange-correlation potential is

$$
\begin{aligned}
v_{x c}^{\left(S_{1}\right)}(\boldsymbol{r}) & =\rho(\boldsymbol{r}) \cdot \delta \epsilon_{x c} / \delta \rho(\boldsymbol{r})+\epsilon_{x c} \\
& =-\frac{E^{\prime}}{N}\left[1+\ln \frac{\rho}{\bar{\rho}}\right]-\frac{1}{2}\left[U_{*}(\boldsymbol{r})+\int \frac{U_{*}(\boldsymbol{r})}{\rho} \mathrm{d} \rho\right] .
\end{aligned}
$$

Another particular solution can be given, if the third term $V_{H}[\rho]$ in eq. (8) is considered to be a constant.

$$
\begin{aligned}
& \epsilon_{x c}^{\left(S_{2}\right)}[\rho]=-\frac{E^{\prime \prime}}{N} \ln \frac{\rho}{\rho}, \\
& E^{\prime \prime}=E_{T}-\sum_{i}^{N} \epsilon_{i}+E_{H}, \\
& E_{H}=V_{H}[\rho]=\frac{1}{2} \int U(\boldsymbol{r}) \rho(\boldsymbol{r}) \mathrm{d} \boldsymbol{r}, \\
& v_{x c}^{\left(S_{2}\right)}(\boldsymbol{r})=-\frac{E^{\prime \prime}}{N}\left[1+\ln \frac{\rho}{\bar{\rho}}\right] .
\end{aligned}
$$

Comparing the two particular solutions given above, it is easy to give the third particular solution when $E^{\prime} \neq E^{\prime \prime}$

$$
\begin{aligned}
& \epsilon_{x c}^{\left(S_{3}\right)}[\rho]=\frac{E^{\prime \prime}}{2\left(E^{\prime}-E^{\prime \prime}\right)} \int \frac{U_{*}(\boldsymbol{r})}{\rho} \mathrm{d} \rho, \\
& v_{x c}^{\left(S_{3}\right)}(\boldsymbol{r})=\frac{E^{\prime \prime}}{2\left(E^{\prime}-E^{\prime \prime}\right)}\left[U_{*}(\boldsymbol{r})+\int \frac{U_{*}(\boldsymbol{r})}{\rho} \mathrm{d} \rho\right] .
\end{aligned}
$$

\subsection{Homogeneous solution $\epsilon_{x c}^{(H)}[\rho]$}

The form of homogeneous solution is assumed to be

$$
\epsilon_{x c}^{(H)}=\sum_{n}\left[c_{n}+d_{n} \ln \rho\right] \rho^{-n / \alpha},
$$

where $\alpha$ is constant, and $c_{n}$ and $d_{n}$ are coefficients related to each other by eq. (10), which can further be simplified to

$$
\begin{aligned}
& c_{n}=\frac{\alpha I\left(1-\frac{n}{\alpha}\right)-n I_{*}\left(1-\frac{n}{\alpha}\right)}{n I\left(1-\frac{n}{\alpha}\right)} d_{n} \quad(n \neq 0), \\
& I(m)=\int \rho^{m} \mathrm{~d} \boldsymbol{r}, \\
& I_{*}(m)=\int \rho^{m} \ln \rho \mathrm{d} \boldsymbol{r} .
\end{aligned}
$$

Note that $d_{0}=0$ and $c_{0}$ could be any arbitrary constant. In fact, a variety of forms could be considered, but will not be discussed in this paper.

\section{Uniform electron gas case}

UEG is actually a model of interacting electrons in a solid where the positive charges are assumed to be uniformly distributed in space whence the electron density is a uniform quantity as well in space. Using a Monte Carlo method, Ceperley and Alder [29] studied this model, and presented the numerical results, which are well described by the PZ/CA parameterization [30]. The exchange functional is derived exactly from the free-electron gas:

$$
\epsilon_{x}=-\frac{p}{r_{s}}, \quad v_{x}=\frac{4}{3} \epsilon_{x},
$$

where $r_{s}=\left(\frac{4 \pi}{3} \rho\right)^{-1 / 3}, p=0.458165$. Parameterization of the correlation functional is slightly more complicated:

$$
\begin{aligned}
& r_{s} \geqslant 1: \\
& \left\{\begin{array}{l}
\epsilon_{c}=g /\left(1+b_{1} r_{s}^{\frac{1}{2}}+b_{2} r_{s}\right), \\
v_{c}=\epsilon_{c} \cdot\left(1+\frac{7}{6} b_{1} r_{s}^{\frac{1}{2}}+\frac{4}{3} b_{2} r_{s}\right) /\left(1+b_{1} r_{s}^{\frac{1}{2}}+b_{2} r_{s}\right),
\end{array}\right. \\
& r_{s}<1: \\
& \left\{\begin{array}{l}
\epsilon_{c}=a \cdot \ln r_{s}+b+c \cdot r_{s} \ln r_{s}+d \cdot r_{s}, \\
v_{c}=a \cdot \ln r_{s}+\left(b-\frac{a}{3}\right)+\frac{2 c}{3} r_{s} \ln r_{s}+\frac{(2 d-c)}{3} r_{s},
\end{array}\right.
\end{aligned}
$$

where $g=-0.1423, b_{1}=1.0529, b_{2}=0.3334, a=0.0311$, $b=-0.048, c=0.0020$ and $d=-0.0116$. Letting $\rho(\boldsymbol{r})=\rho_{c}$, the corresponding $\epsilon_{x c}\left(\rho_{c}\right)$ obtained by the present analysis should be consistent with this exchange-correlation energy density of PZ/CA parameterization. Although the homogenous solution seems to be awkward, when $\rho(\boldsymbol{r})=\rho_{c}$ can be greatly simplified to

$$
\epsilon_{x c}^{(H)}\left[\rho_{c}\right]=c_{0}+\sum_{n \neq 0} \frac{\alpha}{n} d_{n} \rho_{c}^{-\frac{n}{\alpha}} .
$$


In the above expression $\alpha$ is constant, and as requried from here on, we let $\alpha=6$. Besides, $\rho(\boldsymbol{r})=\rho_{c}$ also leads to $\ln \left(\rho_{c} / \bar{\rho}\right)=0$ and $U_{*}(\boldsymbol{r})=0$, which leads to particular solutions $\epsilon_{x c}^{s_{1}}[\rho]=\epsilon_{x c}^{s_{2}}[\rho]=0$ in the case of UEG. Therefore, only the homogenous solution could be used to meet the PZ/CA exchange-correlation energy density. For $r_{s} \geqslant 1$ our exchange-correlation energy density is given by

$$
\begin{aligned}
\epsilon_{x} & =-\frac{p}{r_{s}}, \\
\epsilon_{c} & =c_{0}+\sum_{n=1} \frac{6}{n} d_{n} \rho_{c}^{-\frac{n}{6}} \\
& =c_{0}+\sum_{n=1} \frac{6}{n} d_{n}\left(\frac{3}{4 \pi}\right)^{\frac{n}{6}} r_{s}^{\frac{n}{2}},
\end{aligned}
$$

and the corresponding exchange-correlation potential is

$$
\begin{aligned}
& v_{x}=-\frac{4}{3} \frac{p}{r_{s}} \\
& v_{c}=c_{0}+\sum_{n=1}\left(\frac{6}{n}-1\right) d_{n}\left(\frac{3}{4 \pi}\right)^{\frac{n}{6}} r_{s}^{\frac{n}{2}},
\end{aligned}
$$

where $c_{0}=g$ (the parameter $g=-0.1423$ has been defined in eq. (35)), $d_{1}=-\frac{g \cdot b_{1}^{\prime}}{6}, d_{2}=-2 b_{1}^{\prime} d_{1}-\frac{1}{3} c_{0} b_{2}^{\prime}$, and for $n \geqslant 3$

$$
d_{n}=-b_{1}^{\prime} \frac{n}{n-1} d_{n-1}-b_{2}^{\prime} \frac{n}{n-2} d_{n-2},
$$

where $b_{1}^{\prime}=0.8293$ and $b_{2}^{\prime}=0.2068$. The coefficients given above could be strictly derived by substituting eq. (30) into eqs. (34) and (35), hence for $r_{s} \geqslant 1$ the exchange correlation energy density can be exactly characterized by the homogenous solution. For $r_{s}<1$, the exchange energy density can be obtained in the same way as in $r_{s} \geqslant 1$. However, it is not possible to exactly characterize $\epsilon_{c}$ by the homogenous solution (eq. (30)). This is not surprising, because every term in eq. (30) is required to satisfy the homogenous equation, which is apparently not necessary. Relaxing this requirement, a homogenous solution can be given as:

$$
\begin{aligned}
\epsilon_{c}= & \frac{-2\left(a \cdot \ln r_{\rho}+b+c \cdot r_{\rho} \ln r_{\rho}+d \cdot r_{\rho}\right)^{2}}{J\left[a \cdot \ln r_{\rho}+b+c \cdot r_{\rho} \ln r_{\rho}+d \cdot r_{\rho}\right]} \\
& +2\left(a \cdot \ln r_{\rho}+b+c \cdot r_{\rho} \ln r_{\rho}+d \cdot r_{\rho}\right),
\end{aligned}
$$

where $J[f(\rho)]=\int f(\rho) \mathrm{d} \boldsymbol{r} / \int f(\rho)(\mathrm{d} f(\rho) / \mathrm{d} \rho) \mathrm{d} \boldsymbol{r}$ is functional, and $a, b, c, d$ are the same as in eq. (36), and $r_{\rho}=\left(\frac{4 \pi}{3} \rho(\boldsymbol{r})\right)^{-1 / 3}$ (The symbol $r_{\rho}$ is intended to be distinguished from $r_{s}$, which is usually used when $\rho=\rho_{c}$ ). Note that when $\rho=\rho_{c}$, this homogenous solution is equal to eq. (36), which means that the exchange-correlation energy density, and hence the potential of PZ/CA, can be exactly described by the homogenous solution.

\section{The extension to the non-uniform case}

Having dealt with UEG case, extension to the non-uniform case is of interest, since in reality electron densities are always non-uniform. To extend to the non-uniform case, the homogenous solution adopts PZ/CA exchange-correlation functionals eqs. (35) and (36), and non-uniformity is introduced by adding a particular solution (eq. (29)), which is zero for the uniform case. To be specific, our exchange-correlation functional for the non-uniform case is written as:

$$
\begin{aligned}
& r_{s} \geqslant 1: \\
& \left\{\begin{aligned}
\epsilon_{c}= & g /\left(1+b_{1} r_{s}^{\frac{1}{2}}+b_{2} r_{s}\right)+\beta \int \frac{U_{*}(\boldsymbol{r})}{\rho} \mathrm{d} \rho, \\
v_{c}= & \epsilon_{c} \cdot\left(1+\frac{7}{6} b_{1} r_{s}^{\frac{1}{2}}+\frac{4}{3} b_{2} r_{s}\right) /\left(1+b_{1} r_{s}^{\frac{1}{2}}+b_{2} r_{s}\right) \\
& +\beta\left[U_{*}(\boldsymbol{r})+\int \frac{U_{*}(\boldsymbol{r})}{\rho} \mathrm{d} \rho\right],
\end{aligned}\right. \\
& r_{s}<1: \\
& \left\{\begin{aligned}
\epsilon_{c}= & a \cdot \ln r_{s}+b+c \cdot r_{s} \ln r_{s}+d \cdot r_{s}+\beta \int \frac{U_{*}(\boldsymbol{r})}{\rho} \mathrm{d} \rho, \\
v_{c}= & a \cdot \ln r_{s}+\left(b-\frac{a}{3}\right)+\frac{2 c}{3} r_{s} \ln r_{s}+\frac{(2 d-c)}{3} r_{s} \\
& +\beta\left[U_{*}(\boldsymbol{r})+\int \frac{U_{*}(\boldsymbol{r})}{\rho} \mathrm{d} \rho\right],
\end{aligned}\right.
\end{aligned}
$$

where $\beta=E^{\prime \prime} / 2\left(E^{\prime}-E^{\prime \prime}\right)$ is considered as a material dependent parameter.

\section{Calculations of aluminum and silicon}

Here aluminum and silicon have been used as examples to test the validity of our exchange-correlation functional. Energy variations with respect to the lattice constant are given in Figures 1(c) and (d) (calculations in this paper use ELK code (http://elk.sourceforge.net/, 2014)), which can be fitted by Murnaghan equation of state [34]. One of its popular forms is

$$
E(V)=E_{0}+B_{0} V_{0}\left[\frac{\left(V / V_{0}\right)^{1-B_{0}^{\prime}}}{B_{0}^{\prime}\left(B_{0}^{\prime}-1\right)}+\frac{1}{B_{0}^{\prime}} \frac{V}{V_{0}}-\frac{1}{B_{0}^{\prime}-1}\right],
$$

where $E_{0}, V_{0}$, and $B_{0}$ are energy, volume, and bulk modulus of the system with zero pressure. Bulk modulus $B$ is assumed to be a linear function of pressure $B=B_{0}+P \cdot B_{0}^{\prime}$, where $P=-\mathrm{d} E / \mathrm{d} V$. To fit experimental bulk modulus data, different $\beta$ in eqs. (45) and (46) are used, $\beta=-1.5 \times 10^{-4}$ for aluminum, and $\beta=-0.6 \times 10^{-7}$ for silicon. Comparisons of the lattice constant and the bulk modulus of aluminum and silicon are given in Table 1. It is obvious that GGA (including PW91, PBE, PBEsol) gives a larger lattice constant than local density approximation (i.e. PZ/CA), and PBEsol is closest to the experimental data. However, when one takes bulk modulus into account, existing exchange-correlation functionals could hardly give us a satisfactory answer. Considering the $\beta$ parameter in our exchange-correlation functional, the experimental value of bulk modulus could be fitted, and it is very encouraging to find that the lattice constant is slightly improved after fitting relative to the result from $\mathrm{PZ} / \mathrm{CA}$, which 

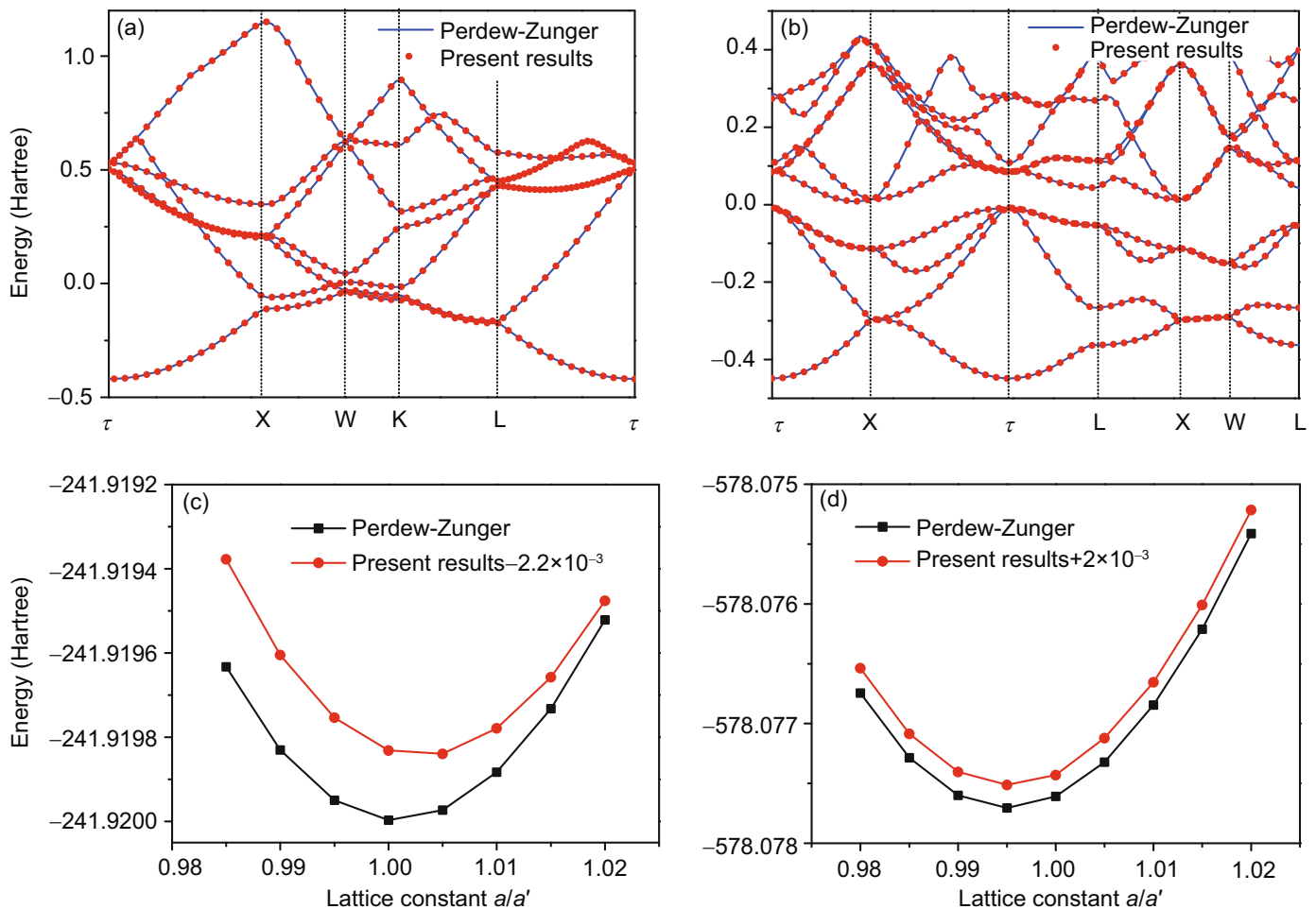

Figure 1 (Color online) Band structure of aluminum (a) and silicon (b) with the PZ/CA exchange-correlation functional (blue solid line) and the current exchange-correlation functional (red scatters) are shown. Energy variation with respect to lattice constant of aluminum (c) and silicon (d) with the PZ/CA exchange-correlation functional and the current exchange-correlation functional are given, where the reference lattice constant $a^{\prime}$ are $3.99 \AA$ and $5.43 \AA$ for aluminum and silicon, respectively. Note that, different $\beta$ in eqs. (45) and (46) are used, $\beta=-1.5 \times 10^{-4}$ for aluminum, and $\beta=-0.6 \times 10^{-7}$ for silicon.

Table 1 Lattice constants and bulk modulus obtained by different exchangecorrelation functionals. The units for lattice constants $a_{0}$ and bulk modulus $B_{0}$ are $\AA$ and $\mathrm{GPa}$, respectively. To fit the bulk modulus, different $\beta$ in eqs. (45) and (46) are used, $\beta=-1.5 \times 10^{-4}$ for aluminum, and $\beta=-0.6 \times 10^{-7}$ for silicon

\begin{tabular}{ccccc}
\hline & $a_{0}(\mathrm{Al})$ & $B_{0}(\mathrm{Al})$ & $a_{0}(\mathrm{Si})$ & $B_{0}(\mathrm{Si})$ \\
\hline Experiments & $4.05[35]$ & $75.9[35]$ & $5.43[36]$ & $98.8[36]$ \\
PZ/CA [30] & 3.99 & 84.3 & 5.40 & 97.7 \\
PW91 [31,32] & 4.08 & 95.3 & 5.47 & 88.6 \\
PBE [11] & 4.05 & 79.9 & 5.47 & 88.8 \\
PBEsol [33] & 4.03 & 82.5 & 5.43 & 94.3 \\
Our results & 4.02 & 75.9 & 5.40 & 98.8 \\
\hline
\end{tabular}

demonstrates the validity of our exchange-correlation functional. Furthermore, the band structure of aluminum and silicon are also given for comparison in Figures 1(a) and (b), from which it can be seen that our results are nearly the same as the results obtained by using the PZ/CA exchangecorrelation functional.

\section{Conclusion}

The total energy equation includes constraints for the exchange-correlation functional, which has been overlooked in previous works. Here we solve the total energy equation for the first time, and provide the formula for the exchangecorrelation functional. In the UEG limit, the formula of exchange-correlation functional exactly returns the results of the jellium model. For the non-uniform electron density case in reality, the particular solution is nonzero and takes the responsibility. Further, calculations of aluminum and silicon are performed. Through tuning $\beta$, our exchangecorrelation functional can predict equilibrium lattice, bulk modulus, and band structure even better than the PZ/CA exchange-correlation functional, which demonstrates its validity. This study, we believe, could provide a new perspective to DFT, and also make it more complete.

We are grateful to Prof. YAO YuGuifor discussions. This work was supported by the National High Technology Research and Development Program of China (Grants Nos. 2014 CB920903 and 2011CBA00100), the National Natural Science Foundation of China (Grant Nos. 11021262, 107212303, 10372107, 11174337 and 11225418), the National Basic Research Program of China (Grants No. 2012CB937500), and the Specialized Research Fund for the Doctoral Program of Higher Education of China (Grants No. 20121101110046).

1 Hohenberg P, Kohn W. Inhomogeneous electron gas. Phys Rev, 1964, 
136: B864-B871

2 Kohn W, Sham L J. Self-consistent equations including exchange and correlation effects. Phys Rev, 1965, 140: A1133-A1138

3 Perdew J P. Density-functional approximation for the correlation energy of the inhomogeneous electron gas. Phys Rev B, 1986, 33: 88228824

4 Becke A D. Density-functional exchange-energy approximation with correct asymptotic behavior. Phys Rev A, 1988, 38: 3098-3100

5 Lee C, Yang W, Parr R G. Development of the Colle-Salvetti correlation-energy formula into a functional of the electron density. Phys Rev B, 1988, 37: 785-798

6 Perdew J P, Wang Y. Accurate and simple analytic representation of the electron-gas correlation energy. Phys Rev B, 1992, 45: 13244-13249

7 Becke A D. A new mixing of Hartree-Fock and local density-functional theories. J Chem Phys, 1993, 98: 1372-1377

8 Gill P. A new gradient-corrected exchange functional. Molecular Phys, 1996, 89: 433-445

9 Becke A D. Density-functional thermochemistry. IV. A new dynamical correlation functional and implications for exact-exchange mixing. J Chem Phys, 1996, 104: 1040-1046

10 Perdew J P, Burke K, Wang Y. Generalized gradient approximation for the exchange-correlation hole of a many-electron system. Phys Rev B, 1996, 54: 16533-16539

11 Perdew J P, Burke K, Ernzerhof M. Generalized gradient approximation made simple. Phys Rev Lett, 1996, 77: 3865-3868

12 Becke A D. Density-functional thermochemistry. V. Systematic optimization of exchange-correlation functionals. J Chem Phys, 1997, 107 : 8554-8560

13 Becke A D. A new inhomogeneity parameter in density-functional theory. J Chem Phys, 1998, 109: 2092-2098

14 Becke A D. Simulation of delocalized exchange by local density functionals. J Chem Phys, 2000, 112: 4020-4026

15 Tao J, Perdew J P, Staroverov V N, et al. Climbing the density functional ladder: nonempirical meta-generalized gradient approximation designed for molecules and solids. Phys Rev Lett, 2003, 91: 146401

16 Becke A D. Real-space post-Hartree-Fock correlation models. J Chem Phys, 2005, 122: 064101

17 Perdew J P, Schmidt K. Jacob's ladder of density functional approximations for the exchange-correlation energy. In: Doren V V, Alsenoy C V, Geerlings P, eds. Density Functional Theory and Its Application to Materials. Antwerp (Belgium): AIP Conference Proceedings, 2001. $1-20$

18 Dykstra C, Frenking G, Kim K, et al. Theory and Application of Computational Chemistry: The First 40 Years. Amsterdam, the Netherlands: Elsevier, 2011. 669-724

19 Oliver G L, Perdew J P. Spin-density gradient expansion for the kinetic energy. Phys Rev A, 1979, 20: 397-403
20 Levy M, Perdew J P. Hellmann-Feynman, virial, and scaling requisites for the exact universal density functionals. Shape of the correlation potential and diamagnetic susceptibility for atoms. Phys Rev A, 1985, 32: 2010-2021

21 Lieb E H, Oxford S. Improved lower bound on the indirect Coulomb energy. Int J Quantum Chem, 1981, 19: 427-439

22 Antoniewicz P R, Kleinman L. Kohn-Sham exchange potential exact to first order in $\rho(\mathrm{K} \rightarrow) / \rho_{0}$. Phys Rev B, 1985, 31: 6779-6781

23 Pan X Y, Sahni V. Criticality of the electron-nucleus cusp condition to local effective potential-energy theories. Phys Rev A, 2003, 67: 012501

24 Levy M. Density-functional exchange correlation through coordinate scaling in adiabatic connection and correlation hole. Phys Rev A, 1991, 43: 4637-4646

25 Levy M, Ou-Yang H. Nonuniform coordinate scaling requirements for exchange-correlation energy. Phys Rev A, 1990, 42: 651-652

26 Pollack L, Perdew J P. Evaluating density functional performance for the quasi-two-dimensional electron gas. J Phys-Condensed Matter, 2000, 12: 1239-1252

27 Gori-Giorgi P, Perdew J P. Pair distribution function of the spinpolarized electron gas: A first-principles analytic model for all uniform densities. Phys Rev B, 2002, 66: 165118

28 Perdew J P, Ruzsinszky A, Tao J, et al. Prescription for the design and selection of density functional approximations: More constraint satisfaction with fewer fits. J Chem Phys, 2005, 123: 062201

29 Ceperley D M, Alder B J. Ground state of the electron gas by a stochastic method. Phys Rev Lett, 1980, 45: 566-569

30 Perdew J P, Zunger A. Self-interaction correction to density-functional approximations for many-electron systems. Phys Rev B, 1981, 23: 5048-5079.

31 Perdew J P, Chevary J A, Vosko S H, et al. Atoms, molecules, solids, and surfaces: Applications of the generalized gradient approximation for exchange and correlation. Phys Rev B, 1992, 46: 6671-6687

32 Perdew J P, Chevary J A, Vosko S H, et al. Erratum: Atoms, molecules, solids, and surfaces: Applications of the generalized gradient approximation for exchange and correlation. Phys Rev B, 1993, 48: 49784978

33 Perdew J P, Ruzsinszky A, Csonka G I, et al. Restoring the densitygradient expansion for exchange in solids and surfaces. Phys Rev Lett, 2008, 100: 136406

34 Murnaghan F. The compressibility of media under extreme pressures. Proc Natl Acad Sci USA, 1944, 30: 244-247

35 Gaudoin R, Foulkes W M C. Ab initio calculations of bulk moduli and comparison with experiment. Phys Rev B, 2002, 66: 052104

36 Beckstein O, Klepeis J, Hart G, et al. First-principles elastic constants and electronic structure of $\alpha-\mathrm{Pt}_{2} \mathrm{Si}$ and PtSi. Phys Rev B, 2001, 63: 134112 